\title{
Can histopathologists reliably assess dysplasia in chronic inflammatory bowel disease?
}

\author{
S A C DUNDAS, ${ }^{*}$ R KAY, $\dagger$ S BECK, D W K COTTON,* A J COUP,§ \\ D N SLATER, $\ddagger$ J C E UNDERWOOD*
}

From the Department of * Pathology, University of Sheffield Medical School, $†$ Probability and Statistics, University of Sheffield, Departments of Histopathology, $\ddagger$ Rotherham District General Hospital, and §Barnsley District General Hospital

SUMMARY A copy of the standardised classification (SC) proposed for assessing dysplasia in inflammatory bowel disease was circulated to six histopathologists who were asked to apply it to 40 slides from 34 patients with ulcerative colitis to test its reproducibility. The slides were relabelled and recirculated to the pathologists at least one month later. Each was asked to state whether or not key diagnostic features were present before giving a final dysplasia score for the second assessment. Only minor interobserver and intraobserver disagreements were recorded. Pathologists were most consistent at recognising back to back glands, villous mucosal architecture, hyperchromatic nuclei, stratification of nuclei, regenerative nuclei and loss of nuclear polarity. There was poor interobserver agreement in assessing dystrophic goblet cells and columnar mucous cells.

Back to back glands, hyperchromatic nuclei, loss of nuclear polarity, stratification of nuclei and columnar mucous cells were considered to be the most important features for determining the severity of dysplasia.

As there was poor interobserver agreement in assessing columnar mucous cells and dystrophic goblet cells these features need to be more clearly defined or should be removed from the SC.

Carcinoma of the colon is a complication of ulcerative colitis. ${ }^{12}$ The risk increases with the duration of the disease and is greater in those with extensive ulcerative colitis. ${ }^{34}$ Retrospective analysis of resected colons from patients with ulcerative colitis and carcinoma show dysplasia in the adjacent or distant mucosa. ${ }^{4-6}$ Colons removed from patients for dysplasia often contain carcinomas. ${ }^{7-9}$ Colonoscopy and biopsy have therefore become essential to the surveillance of carcinoma in ulcerative colitis, but there remain problems with the identification and interpretation of dysplasia, ${ }^{10}$ and studies of observer variation in the recognition of individual criteria are needed. ${ }^{11}$

To overcome the problems of interpretation of dysplasia an international working party was organised to produce a standardised classification of dysplasia and define criteria for its assessment. ${ }^{12}$ This group

Accepted for publication 27 May 1987 jointly produced a proposed classification of dysplasia and assessed its validity in 60 biopsy specimens. Although the group comprised international experts who had previously discussed two slide sets, they found "moderate variability" in the assessment of dysplasia. This may have been due to their selection of a deliberately difficult set of examples for assessment. No attempt was made to assess observer variation of the individual criteria proposed for determination of the grade of dysplasia.

If this standardised classification (SC) is to gain widespread acceptance then the assessment of its validity in the reporting of a representative district general hospital workload is required. Our study was designed to determine how accurately the SC could be applied by a heterogeneous group of histopathologists to a representative slide set. The study was also designed to determine the interobserver variation of the proposed criteria and how each pathologist weighed them in determining the grade of dysplasia. 


\section{Material and methods}

All the pathology reports on rectal and colonic biopsy specimens between 1980 and 1986 from patients with chronic inflammatory bowel disease were examined to identify those cases in which the presence of dysplasia had been mentioned. The slides from these 62 cases were examined by one observer who selected 40 slides. The 40 slides had been made of 40 rectal or colonic biopsy specimens from 34 patients $(17$ men and 17 women; mean age 48, range 23-86 years). Thirty patients did not have carcinoma (mean 12 years, range one to 30 ), four did have carcinoma (mean 16 years, range four to 26). The slides showed a spectrum of dysplastic changes from inactive colitis (negative for dysplasia) to high grade dysplasia according to the criteria of Riddell et al. ${ }^{12}$ The notes and pathological reports on each of the 34 patients from which the slides were selected were reviewed. Eight slides were selected from four patients who subsequently had colectomies carried out for colonic carcinoma.

Six histopathologists - two with a specialised interest in gastroenterology, two with a specialised interest in dermatopathology, and two district general hospital pathologists - took part in the study. All except one had passed the final MRCPath examination, and experience varied between four and 20 years.

Each pathologist was given a copy of the SC proposed by Riddell et al ${ }^{12}$ at least two weeks before receiving the slides. They were instructed to read the SC paper and then grade each slide according to the proposed criteria on a numerical scale of $0-7$ (table 1) (assessment 1 ). The slides were randomised by a third party, relabelled, and recirculated to each pathologist at least one month later. On the second assessment the pathologists were asked to state whether or not the SC features were present in the "most dysplastic" glands to any significant degree in every slide and then score each slide on the same 0-7 scale.

Intraobserver variability was assessed by calculating the rank of correlation coefficients between the two assessments for each observer. Random effects analysis of variance ${ }^{13}$ was used to measure the variability between assessors on each of the two assess-

Table 1 Score allocation

\begin{tabular}{ll}
\hline Biopsy classification & Numerical score \\
\hline Normal mucosa & 0 \\
Inactive colitis & 1 \\
Active colitis (no dysplasia) & 2 \\
Probably negative & 3 \\
Unknown & 4 \\
Probably positive & 5 \\
Low grade dysplasia & 6 \\
High grade dysplasia & 7 \\
\hline
\end{tabular}

ments. Variability in this case was measured by the standard deviation attributable to measurements by different pathologists. The mean \pm 2 standard deviations contained roughly $95 \%$ of pathologists' scores for any particular slide. Agreement scores for each feature in the second assessment were calculated as average scores over slides. The score for each slide was defined as the greater number of affirmatives and negatives across the six assessors. The features looked for were: regenerative nuclei; loss of polarity; hyperchromatic nuclei; tightly packed crypts; dystrophic goblet cells; stratified nuclei; villous pattern; columnar mucous cells; back to back glands.

The importance of each feature in the assessment for each slide was determined by modelling the overall score on the presence or absence of each feature in a regression model. The model appropriate for this was the ordinal logistic model. ${ }^{14}$ This allows the (partial) correlation of each feature with the score to be measured; rankings of the importance of these features can then be based on the magnitude of the correlation.

\section{Results}

\section{CASE REPORTS}

Case 1 was a woman whose colitis had begun at 43 years of age; 14 years later she was referred to our hospital with a right iliac fossa mass, and radiological investigations showed a possible carcinoma. A rectal biopsy specimen showed chronic ulcerative colitis and moderate dysplasia (mean study score $5 \cdot 1$ ). The colectomy specimen showed Dukes' grade C adenocarcinoma; she died 14 months later with disseminated adenocarcinoma.

Case 2 was a man whose colitis had begun at 55 . His first colonic examination was carried out 14 years later following the onset of anaemia. Biopsy specimens showed chronic ulcerative colitis to the hepatic flexure and severe dysplasia (mean study score 6.8). A colectomy was carried out and this showed Dukes' A adenocarcinoma.

Case 3 was a woman whose colitis had begun at 27. She had intermittent colitis managed with salazopyrin, prednisolone enemas, and oral prednisolone. Early rectal and colonoscopic biopsy specimens showed ulcerative colitis with no or moderate dysplasia. Severe dysplasia (mean study score 6.3) was first reported 18 years after the onset of colitis. A colectomy carried out two years later (delayed due to patient resistance) showed Dukes' $B$ adenocarcinoma. At the time of writing she was being treated for ovarian and liver metastases.

Case 4 was a man whose colitis had begun at 50 . A rectal biopsy performed one year after the onset of colitis showed moderate dysplasia (mean study score 
$4 \cdot 1$; this was followed by multiple rectal and colonoscopic biopsies. The specimens showed moderate (mean study score 5.7), mild (mean study score 5.0), severe (mean study score 5.7 ) and severe (mean study score 5.8 ) dysplasia. The colectomy performed one year after the first report of dysplasia showed Dukes' $B$ adenocarcinoma.

\section{OBSER VER VARIABILITY \\ Intraobserver variability}

Table 2 shows the rank correlation coefficient of the six pathologists who showed significant ability to rank the biopsy specimens into grades of dysplasia ( $p$ $<0.001$ in a test of zero correlation for each pathologist). The two pathologists with a special interest in gastrointestinal disease were not significantly more consistent than non-specialists.

\section{Interobserver variability}

A random effects analysis of variance with the standard deviations between assessors produced a score of 0.66 for assessment 1 and 0.83 for assessment 2 . Taking 2 standard deviations from the mean in assessment $1,95 \%$ of assessors would give a score within 1.3 of the mean for all assessments.

Interobserver variability was greater when the pathologists were asked specifically to state the presence or absence of key features (assessment 2).To determine the reason for this the agreement scores for each feature (table 3) and ranking of the importance of each feature were calculated. The agreement scores show that pathologists were most consistent in recognising back to back glands and villous pattern (on average more than five of six assessors in agreement (table 3). The ranking order of the nine features assessed shows that back to back glands, hyperchromatic nuclei, loss of polarity, stratified nuclei and columnar mucous cells were significantly associated (in a positive way) with the final score while the remainder were not. These two analyses show that back to back glands were considered to be the most important feature in determining the degree of dysplasia and they were also consistently recognised. In contrast, columnar mucous cells (least agreement) were

Table 2 Rank correlation coefficients in decreasing order between both assessments for six pathologists

\begin{tabular}{ll}
\hline Pathologist & Correlation coefficient \\
\hline 1 & 0.95 \\
2 & 0.76 \\
3 & 0.71 \\
4 & 0.69 \\
5 & 0.65 \\
6 & 0.61 \\
\hline
\end{tabular}

Large values (close to 1) indicate excellent agreement (consistency) between the two assessments.
Table 3 Agreement scores

\begin{tabular}{lc}
\hline Feature & Score \\
\hline Back to back glands & $5 \cdot 5^{*}$ \\
Villous pattern & $5 \cdot 1$ \\
Hyperchromatic nuclei & $4 \cdot 7$ \\
Stratification of nuclei & $4 \cdot 7$ \\
Tightly packed crypts & $4 \cdot 6$ \\
Regenerative nuclei & $4 \cdot 6$ \\
Loss of polarity & $4 \cdot 6$ \\
Dystrophic goblet cells & $4 \cdot 3$ \\
Columnar mucous cells & $4 \cdot 0$ \\
\hline
\end{tabular}

Scores above 5 indicate that on average more than five of six assessors were in agreement. A score of 3 would represent total disagreement-that is, three pathologists think a feature is present and three do not.

considered to be important in grading dysplasia but there was little agreement between pathologists as to whether or not they were present. This interobserver disagreement in determining the presence or absence of columnar mucous cells (and to a lesser degree loss of nuclear polarity) may have accounted for the greater disagreement in the second assessment.

\section{CLINICAL RELEVANCE OF GRADING}

To assess the likely clinical outcome of any variability in grading the average grade for each biopsy specimen in the first assessment was calculated. These average grades were divided into four categories: those negative or probably negative for dysplasia (grade 0-3.49) those probably unknown or probably positive for dysplasia (3.5-5.49); low grade dysplasia (5.5-6.25); and high grade dysplasia (6.26-7). For each category of biopsy specimen the total number of times that it was scored correctly or incorrectly in some other category was calculated (table 4).

Table 4 shows that there were 150 readings of biopsy specimens with a mean score of 3.49 or less (negative or probably negative for dysplasia). Of these, on 112 occasions the biopsy specimens were correctly read as negative or probably negative for dysplasia. There were 32 readings assigning specimens to the unknown or probably positive category. There were six false readings of low grade dysplasia which clinically would result in a repeat biopsy. There were no false readings of high grade dysplasia which would result in inappropriate colectomy.

There were 30 readings of biopsy specimens as high grade dysplasia. On 21 occasions the biopsy specimens were correctly read. There were nine false negative readings, assigning specimens to the probably negative, indefinite or low grade dysplasia category; this would result clinically in a repeat biopsy. There were no false readings assigning biopsy specimens from the high grade dysplasia category into the negative group. 
Table 4 Clinical relevance of grading

\begin{tabular}{|c|c|c|c|c|c|}
\hline \multirow[b]{2}{*}{ Average grade } & \multicolumn{4}{|c|}{ Assigned grades for dysplasia } & \multirow[b]{2}{*}{ Total } \\
\hline & $\begin{array}{l}\text { Negative or } \\
\text { probably negative }\end{array}$ & $\begin{array}{l}\text { Unknown or } \\
\text { probably positive }\end{array}$ & Low grade & High grade & \\
\hline \multirow{2}{*}{$\begin{array}{l}0-3 \cdot 49 \text { (negative or probably } \\
\text { negative) } \\
3 \cdot 50-5 \cdot 49 \text { (unknown or probably } \\
\text { positive) } \\
5 \cdot 50-6 \cdot 25 \text { (low grade dysplasia) } \\
6 \cdot 26-7 \text { (high grade dysplasia) }\end{array}$} & 112 & 32 & 6 & 0 & 150 \\
\hline & $\begin{array}{r}11 \\
2 \\
0\end{array}$ & $\begin{array}{r}14 \\
7 \\
4\end{array}$ & $\begin{array}{r}10 \\
11 \\
5\end{array}$ & $\begin{array}{r}1 \\
4 \\
21\end{array}$ & $\begin{array}{l}36 \\
24 \\
30\end{array}$ \\
\hline
\end{tabular}

\section{Discussion}

The formation of the dysplasia morphology study group was a welcome and long overdue attempt to combine the expertise of a group of international experts to produce a standardised classification (SC) for dysplasia in inflammatory bowel disease. ${ }^{12}$ To assess intraobserver and interobserver variability 60 biopsy specimens were reviewed by 10 pathologists according to the SC criteria.

Riddell et al calculated a "discrepancy score" to assess the intraobserver and interobserver variability. To directly compare our results with those of the SC group we repeated their method (table 5). Two conclusions can be drawn: firstly, the mean intraobserver variability in our study ( 0.4 grade units) was only marginally greater than that of the SC study group (0.29 grade units); secondly, the interobserver variability in our group was similar to that of the SC group ( 0.6 and 0.58 grade units). This contrasts with the poor intraobserver and interobserver variations in the degree of differentiation of rectal carcinoma reported by Thomas et $a l^{15}$ and clearly shows that the $\mathrm{SC}$ is valid and can be reproduced by non-specialist pathologists.

The discrepancy score analysis used by Riddell $e t$ al causes a "statistical" reduction in the mean intraobserver and interobserver variability at either end of the numerical scale-that is, low and high grade lesions. This is because a biopsy specimen with a true grade of 3 can only be scored as high as 3 (not 3.5 ) whereas a biopsy specimen in the middle grades such as 2 may be scored between 1.5 and 2.5 .

Analysis of our results by random effects analysis and by the method of Riddell et al (table 5) shows a greater interobserver variability in the second assessment (in which pathologists had to state whether or not specific features were present) than the first. This is probably because pathologists in our study thought that columnar mucous cells were important in determining the grade of dysplasia, but there was almost no agreement as to whether or not these cells were present (table 3).

Examination of the case notes of the 34 patients included in the study showed that four patients subsequently developed carcinoma, one of these had metastatic carcinoma and another died from metastatic spread. Two of the four patients with carcinoma in our study were not referred for colonoscopic surveillance. Presumably the general practitioner of the first patient and the consultant in charge of the other felt that screening for dysplasia was either not indicated or not useful. Both of the other patients had histological reports of severe dysplasia nine months and two years before colectomy was carried out. This delay between the first reports of dysplasia and colectomy suggest that even when severe dysplasia is reported clinicians are not sufficiently confident in the histological diagnosis to proceed to early colectomy.

A recent editorial in the Lancet highlighted the fact that screening programmes for carcinoma in ulcerative colitis do fail ${ }^{10}$ and that "there are difficulties in the histological interpretation of dysplasia (probably

Table 5 Discrepancy score

\begin{tabular}{|c|c|c|c|c|c|}
\hline \multicolumn{3}{|l|}{ Average grade } & \multicolumn{3}{|c|}{ Mean variations } \\
\hline (Our scoring system) & Riddell et al & Equivalent grade $(n=)$ & Intra & Inter I & Inter 2 \\
\hline \multirow[t]{2}{*}{$\begin{array}{l}(0-3 \cdot 49) \\
(3 \cdot 50-5 \cdot 49) \\
(5 \cdot 50-6 \cdot 25) \\
(6 \cdot 26-7)\end{array}$} & $\begin{array}{l}0-0.74 \\
0.75-1.49 \\
1.5-2.24 \\
2.25-3.0\end{array}$ & $\begin{array}{r}25 \\
6 \\
4 \\
5\end{array}$ & $\begin{array}{l}0.31 \\
0.58 \\
0.67 \\
0.47\end{array}$ & $\begin{array}{l}0.44 \\
0.86 \\
1.02 \\
0.63\end{array}$ & $\begin{array}{l}0.61 \\
1.13 \\
1 \cdot 28 \\
0.60\end{array}$ \\
\hline & & 40 & 0.40 & 0.58 & 0.76 \\
\hline
\end{tabular}


lessened by the report of an international working party)". Clearly, if clinicians are going to carry out colectomies for histological dysplasia they need to be confident of our ability to diagnose it. In our study the five biopsy specimens with high grade dysplasia were scored as such on 21 of 30 readings (table 4). Although in nine other readings lower grades were assigned, there were no readings in which dysplasia was totally missed. In 150 readings of the 25 biopsy specimens that were negative or unknown for dysplasia there were no false readings of high grade dysplasia (table 4); in six readings low grade dysplasia was diagnosed but clinically this would only result in rebiopsy.

We have shown that the SC proposed by Riddell et al can be reliably applied to colonoscopic biopsy specimens by a group of histopathologists of different special interest. There is still a minor degree of difference in opinion between experts and the nonexperts in our study. This may be improved by more precise definition of features such as "columnar mucous cells" and "dystrophic goblet cells"; alternatively, these features should not be included in the SC.

\section{References}

1 Goldgraber MB, Kirsner JB. Carcinoma of the colon in ulcerative colitis. Cancer 1964;17:657-65.

2 Katza I, Brady RS, Morris E, Katz S. Assessment of colorectal cancer risk in patients with ulcerative colitis: experience from a private practice. Gastroenterology 1983;85:22-9.

3 Derroede GJ, Taylor WF, Sauer WG. Cancer risk and life expectancy of children with ulcerative colitis. $N$ Engl $J$ Med 1971;285:17-21.

4 Morson BC, Pang SC. Rectal biopsy as an aid to cancer control in ulcerative colitis. Gut 1962;8:423-34.

5 Cook MG, Goligher JC. Carcinoma and epithelial dysplasia complicating ulcerative colitis. Gastroenterology 1975;68: $1127-36$.

6 Yardley JH, Keren DF. "Pre-cancer" lesions in ulcerative colitis: a retrospective study of rectal biopsy and colectomy specimens. Cancer 1974;34:835-44.

7 Rosenstock E, Farmer RG, Petras R, Swak MV, Rankin GB, Sullivan BH. Surveillance for colonic carcinoma in ulcerative colitis. Gastroenterology 1985;89:1342-6.

8 Lennard-Jones JE, Morson BC, Ritchie JK, Williams CB. Cancer surveillance in ulcerative colitis. Lancet 1983;ii:149-52.

9 Dickinson RJ, Dixon MF, Axon ATR. Colonoscopy and the detection of dysplasia in patients with longstanding ulcerative colitis. Lancet 1980;ii:620-2.

10 Anonymous. Colrectal carcinoma in ulcerative colitis. [Editorial] Lancet 1986;ii:197-8.

11 Lennard Jones JE, Morson BC, Ritchie JK, Shore DC, Williams CB. Cancer in colitis: assessment of the individual risk by clinical and histological criteria. Gastroenterology 1977;73:1280-9.

12 Riddell RH, Goldman H, Ransohoff DF, et al. Dysplasia in inflammatory bowel disease: standardized classification with provisional clinical applications. Hum Pathol 1983;14:931-67.

13 Searle SR. Linear models. New York: John Wiley and Sons, 1971.

14 McCullagh P. Regression models for ordinal data. J Roy Statist Soc B 1980;42:109-42.

15 Thomas GDH, Dixon MF, Smeeton NC, Williams NS. Observer variation in the histological grading of rectal carcinoma. J Clin Pathol 1983;36:385-91.

Requests for reprints to: Dr SAC Dundas,.Department of Pathology, University of Sheffield Medical School, Beechō Hill Road, Sheffield S10 2RX, England. 